\title{
Determining the cohesive energy of coronene by dispersion-corrected DFT methods: Periodic boundary conditions vs. molecular pairs
}

\author{
J. C. Sancho-García, ${ }^{1, a)}$ A. J. Pérez-Jiménez,, ${ }^{1, b)}$ and Y. Olivier ${ }^{2}$ \\ ${ }^{1}$ Departamento de Química Física, Universidad de Alicante, E-03080 Alicante, Spain \\ ${ }^{2}$ Laboratory for Chemistry of Novel Materials, University of Mons, B-7000 Mons, Belgium
}

(Received 25 November 2014; accepted 16 January 2015; published online 3 February 2015)

\begin{abstract}
We investigate the cohesive energy of crystalline coronene by the dispersion-corrected methods DFT-D2, DFT-D3, and DFT-NL. For that purpose, we first employ bulk periodic boundary conditions and carefully analyze next all the interacting pairs of molecules within the crystalline structure. Our calculations reveal the nature and importance of the binding forces in every molecular pair tackled and provide revised estimates of the effects of two- and three-body terms, leading to accurate results in close agreement with experimental (sublimation enthalpies) reference values. (O) 2015 AIP Publishing LLC. [http://dx.doi.org/10.1063/1.4907268]
\end{abstract}

\section{INTRODUCTION}

The coronene molecule $\left(\mathrm{C}_{24} \mathrm{H}_{12}\right.$, see Figure 1) crystallizes in the monoclinic pattern with all the intermolecular separations corresponding to van der Waals distances. These molecules pack making the most efficient use of space, keeping a compromise between through-space attractive and repulsive forces. This gives rise to what is known as $\gamma$ motif, with molecules arranging in nearly parallel directions to facilitate overlap between $\pi$-orbitals, although a slipped T-shape orientation between columns is also forced. ${ }^{1,2}$

The electronic properties of these molecular solids are often highly anisotropic in nature, which reflects different orientations found for the molecules in the solid formed, but close to those in solution, which reflects the weak interactions existing between the molecules. Most importantly, these solids form soft and brittle crystals, and are thus able to be incorporated in multi-layered and advanced organic electronic devices, as far as their stability and supramolecular order is completely understood and controlled. The latter properties are completely driven in apolar molecules by dispersion interactions, arising from instantaneous electronic fluctuations not attributed to any permanent electric multipole, which is known to be a highly challenging research topic for today's calculations. ${ }^{3-8}$

Actually, a benchmark set for non-covalent interactions in solids, the X23 dataset including sublimation energies of 23 organic crystals, ${ }^{9,10}$ has revealed as the modern and most accurate framework to assess a variety of theoretical methods. ${ }^{11,12}$ The set includes diverse cases, chemically speaking, ranging from small (ammonia, acetic acid, formamide, and cyanamide, to name just a few examples) to larger molecules, such as (nonpolar) benzene, naphthalene, anthracene, or adamantane, or (polar) aza-substituted compounds (imidazole, pyrazine, pyrazole, hexamine, and triazine) or biomolecules (urea, uracil, and cytosine). The application of Density Functional Theory

\footnotetext{
a)E-mail: jc.sancho@ua.es

b)E-mail: aj.perez@ua.es
}

(DFT) to the X23 dataset, necessarily with some correction for dispersion, was found to provide very accurate values showing mean absolute deviations of 1-2 kcal/mol compared to experimental reference values, ${ }^{13}$ and thus very close to the uncertainties of the latter. Such accuracy might pave the way towards optimizing crystal structure parameters ${ }^{14,15}$ or disclosing polymorphism issues, ${ }^{16,17}$ although with due caution in very challenging cases, such as the quasi-degeneracy in crystalline aspirin, ${ }^{18,19}$ oxalyl dihydrazide,${ }^{20}$ or para-diiodobenzene, ${ }^{21}$ to mention some recently reported examples.

By focusing on crystalline coronene, we aim at contributing to recent efforts ${ }^{22-29}$ that attempted to disentangle the importance of the underlying forces involved in the calculation of lattice or cohesive energies. This molecule is not included in the aforesaid X23 dataset, but it is an excellent prototype of the kind of interactions found between graphene layers. As a matter of example of the interest on this and closely related molecules, we mention the recent modeling of the intrusion of molecules into graphite using coronene clusters as model for extended graphene. ${ }^{30}$ Very recently too, a quantitative relationship between lattice structure and band gap of polycyclic aromatic hydrocarbons, including coronene, was also theoretically investigated. ${ }^{31}$ We also mention the benchmarking of the (long-range corrected) $\omega \mathrm{B} 97 \mathrm{X}-\mathrm{V}$ functional against the binding energy of the parallel-displaced coronene dimer, in order to assure that the functional could be applied to larger systems out of the training set. ${ }^{32}$ This dimer has been historically considered a cost-efficient model to study a number of key properties of more extended systems, such as the interlayer interactions in graphite, ${ }^{33}$ the hydrogen adsorption and storage in layered materials, ${ }^{34}$ or the influence of electroactive substituents on the long-range order in nanosamples. ${ }^{35}$ To do this in a systematic fashion, we will follow a top-down or decomposition approach, starting first from unit cell calculations using periodic boundary conditions, trying in a second step to gain insight by considering its compositional sub-systems (pairs of interacting molecules) rigidly extracted from the crystalline structure. 


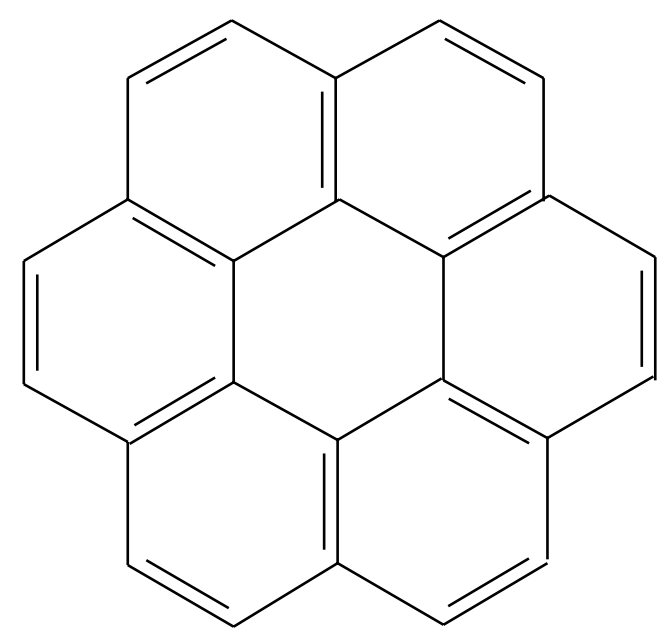

FIG. 1. Chemical structure of coronene, omitting the hydrogen atoms and corresponding $\mathrm{C}-\mathrm{H}$ bonds for clarity.

\section{REFERENCE VALUES}

The calculated cohesive energies, $E_{\text {cohesive }}$, using the two strategies underlined above will be compared (when needed) to experimental sublimation energies, $\Delta_{S} H(T)$, at room temperature (298.15 K) by adding the contribution $2 R T,{ }^{36,37}$ and thus obtaining $E_{\text {cohesive }}=\Delta_{s} H(T)+2 R T$, which will also allow us to compare with previous (although very scarce) theoretical estimates from the literature. Note also the large uncertainties (of the order of 3-9 kJ/mol) affecting the experimental available $\Delta_{s} H$ values for coronene; therefore, one must rely on averaged values and reported error bars from different measurements, leading to a modern estimate at room temperature of $\Delta_{s} H=142.6 \pm 8.7 \mathrm{~kJ} / \mathrm{mol} .{ }^{38}$ Additionally, it is also known that the sublimation enthalpy of coronene should exceed by at least few $\mathrm{kJ} / \mathrm{mol}$ its vaporization enthalpy, which is $\Delta_{v} H$ $=139.5 \pm 6.0 \mathrm{~kJ} / \mathrm{mol}$ according to Ref. 38 too. Note that the final value of $E_{\text {cohesive }}$ is made positive to compare with experimental data on sublimation energies.

The crystal structure was obtained from the Crystallography Open Database (code archive 9009975) ${ }^{39}$ and used without further modification. The interacting dimers were rigidly extracted from it. The MERCURY program ${ }^{40}$ was employed for visualizing and manipulating crystal structures in three dimensions.

\section{CALCULATIONS USING PERIODIC BOUNDARY CONDITIONS}

The cohesive energy of crystalline coronene (see Figure 2 ) is usually estimated from the bulk as ${ }^{41,42}$

$$
E_{\text {cohesive }}=\frac{E_{\text {bulk }}}{Z}-E_{\text {molecule }},
$$

where $E_{b u l k}$ is the total energy of the unit cell $(a=16.11 \AA$, $b=4.70 \AA, c=10.10 \AA, \alpha=\gamma=90^{\circ}, \beta=110.9^{\circ}$ ) including $Z$ molecules, and $E_{\text {molecule }}$ the energy of an isolated molecule, both energies calculated here at the dispersion-corrected DFTD2 level. The missing dispersion energy $\left(E_{\mathrm{D}}\right)$ of uncorrected DFT calculation is efficiently added, as implemented in the

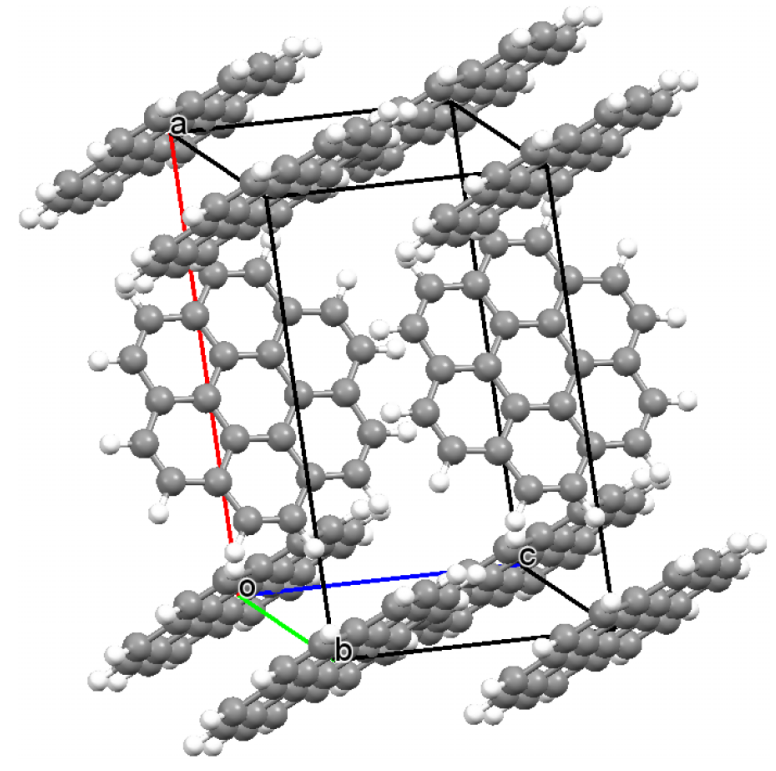

FIG. 2. Unit cell structure of coronene, showing the crystallographic a, b, and $\mathrm{c}$ axes.

package ABINIT, ${ }^{43-45}$ by the -D2 function ${ }^{46,47}$

$$
E_{\mathrm{D} 2}=-s_{6} \sum_{B>A}^{N} \frac{C_{6}^{A B}}{R_{A B}^{6}} f\left(R_{A B}\right),
$$

where $A$ and $B$ are the atom labels, separated thus by the distance $R_{A B}=\left|R_{A}-R_{B}\right|$, of the weakly interacting molecules, $C_{6}^{A B}$ are the interatomic dispersion coefficients, and $f\left(R_{A B}\right)$ is a damping function for ensuring a proper short- to longrange transition of the dispersion energy. The parameter $s_{6}$ is thus adapted to any underlying density functional in use, Perdew-Burke-Ernzerhof (PBE) ${ }^{48}$ and Becke-Lee-Yang-Parr $(B L Y P)^{49,50}$ here (see Table I), coupling both energy (electronic and dispersion) terms. Other technical details for these calculations are underlined next: (i) we sample the Brillouin zone by the Monkhorst-Pack scheme with a $k$-grid point of $2 \times 6 \times 3$, which was sufficiently converged with respect to the corresponding $4 \times 12 \times 6$ grid; (ii) we fix a cutoff energy of plane waves at $100 \mathrm{Ry}$ after checking for convergence of values up to $0.1 \mathrm{~kJ} / \mathrm{mol}$ or less; and (iii) the monomer energy was also carefully converged by employing a cell of $35 \times 35 \times 30 \AA$ alongside the $x, y$, and $z$ directions, respectively. Furthermore, for the BLYP-D2 calculations, a norm-conserving Martins-

TABLE I. List of parameters entering into the (functional-specific) dispersion correction used.

\begin{tabular}{lccccc}
\hline \hline Method & $s_{6}$ & $s_{r, 6}$ & $s_{8}$ & $s_{r, 8}$ & $b$ \\
\hline BLYP-D2 & 1.20 & $\ldots$ & $\ldots$ & $\ldots$ & $\ldots$ \\
PBE-D2 & 0.75 & $\ldots$ & $\ldots$ & $\ldots$ & $\ldots$ \\
BLYP-D3 & 1.000 & 1.094 & 1.692 & 1.000 & $\ldots$ \\
B3LYP-D3 & 1.000 & 1.261 & 1.703 & 1.000 & $\ldots$ \\
B2-PLYP-D3 & 0.640 & 1.427 & 1.022 & 1.000 & $\ldots$ \\
BLYP-NL & $\ldots$ & $\ldots$ & $\ldots$ & $\ldots$ & 4.0 \\
B3LYP-NL & $\ldots$ & $\ldots$ & $\ldots$ & $\ldots$ & 4.8 \\
B2-PLYP-NL & $\ldots$ & $\ldots$ & $\ldots$ & $\ldots$ & 8.0 \\
\hline \hline
\end{tabular}


Trouiller (MT) pseudopotential was used as adapted from the extended Car-Parrinello (CP) molecular dynamics pseudolibrary (http://cpmd.org), denoted thus as BLYP-D2/MTCP. As a sanity check, for the PBE-D2 calculations, we also compare the latter method (PBE-D2/MTCP) with the default MT pseudopotentials implemented in the code used (PBED2/MTAB) to see if any difference due to the use of different pseudopotentials in different codes might largely influence the results or not.

Our PBE-D2/MTAB, PBE-D2/MTCP, and BLYP-D2/ MTCP results for $E_{\text {cohesive }}$ are $134.4,133.1$, and $162.4 \mathrm{~kJ} / \mathrm{mol}$, respectively, which shows the little influence of standards pseudopotentials (compare PBE-D2/MTAB and PBED2/MTCP) once due cautions for compatibility between the different formats are taken. However, what seems to have a more pronounced effect is the use of the PBE or the BLYP functional (compare PBE-D2/MTCP and BLYP-D2/MTCP). Whereas the former slightly underestimates the (averaged) experimental value by up to $14 \mathrm{~kJ} / \mathrm{mol}$, although agrees well with other estimates in the literature using the same PBE-D2 scheme, ${ }^{51}$ the latter overestimates the experimental value by around $15 \mathrm{~kJ} / \mathrm{mol}$. These values are thus, broadly speaking, under- or overestimating the experimental value by around $3 \mathrm{kcal} / \mathrm{mol}$. However, the often claimed "chemical accuracy" (an error bar with respect to reference values of about $1 \mathrm{kcal} / \mathrm{mol}$ ) is unrealistic for large systems, and $2-3 \mathrm{kcal} / \mathrm{mol}$ is nowadays considered as a more realistic target. ${ }^{52}$ Despite this, since disclosing of polymorphism issues would need a greater accuracy, and reaching this stage, one might thus ask if the density functional and/or the dispersion-correction are behind the errors found.

To further analyze and complete these results, trying to grant some physical insights into the reasons why one functional behaves (slightly) different than another, we know that the exchange functional in BLYP is known to be much more repulsive for non-covalent interactions than the exchange functional of PBE, ${ }^{53}$ and thus BLYP always benefits more from the (attractive) dispersion corrections, which can partly explain its overestimation. Furthermore, we have only included here mostly dipole-dipole, scaling as $C_{6} R^{-6}$ through the use of Eq. (2) interactions, neglecting dipole-quadrupole forces scaling as $C_{8} R^{-8}$, which might also play a significant role as found before for other related polycyclic aromatic hydrocarbons. ${ }^{54,55}$ Finally, we would like to also assess the influence of higherorder terms beyond pair interactions (e.g., interactions between all atom trimers) in the dispersion correction used, as it was recently done for benzene lattice or cohesive energy. ${ }^{56}$ All these issues will thus constitute the guide for the next part of the study.

\section{CALCULATIONS USING SUM OF ENERGIES OF INTERACTING MOLECULAR PAIRS}

The energy, $\Delta E_{\text {int }}$, between two or more weakly interacting $P, Q, R$. . molecules or fragments, responsible for their self-assembly, is difficult to evaluate directly and depends on many-body electronic interactions. ${ }^{57,58}$ Thus, for computational efficiency and for insightful physical interpretations, it is worthwhile to decompose it into contributions from dimers, trimers, and so on. This leads to an accurate decomposition, known as the many-body expansion

$\Delta E_{\text {int }}=\sum_{P<Q} \Delta E_{\text {int }}(P Q)+\sum_{P<Q<R} \Delta E_{\text {int }}(P Q R)+\cdots$,

where $\Delta E_{\text {int }}(P Q)$ is the two-body contribution, $\Delta E_{\text {int }}(P Q R)$ is the three-body contribution, and the like. The expansion converges quickly, for the benzene case, the contribution from tetramers was less than $1 \%$ of the final theoretical value, ${ }^{56}$ and is normally truncated after the first two terms, which are defined as

$$
\begin{gathered}
\Delta E_{\text {int }}(P Q)=E(P Q)-E(P)-E(Q), \\
\Delta E_{\text {int }}(P Q R)=E(P Q R)-E(P Q)-E(P R)-E(Q R),
\end{gathered}
$$

where $E(P Q R)$ and $E(P Q)$ are the energies of the supermolecular complex formed by two or three molecules, respectively, and $E(P)$ or $E(Q)$ the energy of the isolated monomer. The key is to find appropriate expressions for calculating the $E(P Q)$ energies, and a good approximation (cost-efficient) for the three-body term $\triangle E_{\text {int }}(P Q R)$, which normally arises from the effects of polarization in the aggregate of molecules. We will briefly explain next how to introduce the dispersion energy $\left(E_{\mathrm{D}}\right)$ for any (intended) accurate calculation of $\Delta E_{\text {int }}(P Q)$, and how this also allows to approximately introduce three-body effects according to available current theories.

\section{A. Theoretical details}

\section{The DFT-D3 method}

First, for efficiently incorporating the dispersion energy to any calculation of $E(P Q)$, we will use the recent extension of Eq. (2), coined as DFT-D3, ${ }^{59}$ in which this missing energy is given now through the refined function

$$
E_{\mathrm{D} 3}(A, B)=-\sum_{n=6,8} s_{n} \sum_{B>A}^{\text {atom pairs }} \frac{C_{n}^{A B}}{R_{A B}^{n}} f_{n}\left(R_{A B}\right),
$$

where $A$ and $B$ represent atoms of the (weakly) interacting molecules, separated by the distance $R_{A B}$, but the sum extends now to the dipole-quadrupole term and depends on the $n$ th-order interatomic dispersion coefficients $C_{n}^{A B}$ and some functional-dependent parameters $s_{n}$. One then damps the dispersion contribution to zero at short distances, the ZeroDamping (ZD) scheme

$$
f_{n}\left(R_{A B}\right)=\frac{1}{1+6\left(\frac{R_{A B}}{s_{r, n} R_{A B}^{0}}\right)^{-\alpha}},
$$

with $R_{A B}^{0}=\sqrt{\frac{C_{8}^{A B}}{C_{6}^{A B}}}$. More information about the role played by damping functions may be found in Refs. 60-62. Using the DFT-D3 method, it is also possible to approximate three-body effects through the function ${ }^{63}$

$$
\begin{aligned}
E_{\mathrm{D} 3}(A, B, C) & \\
= & \sum_{C>B>A}^{\text {atom triples }} C_{9}^{A B C} \frac{\left(3 \cos \theta_{A B} \cos \theta_{B C} \cos \theta_{A C}+1\right)}{\left(R_{A B} R_{B C} R_{A C}\right)^{3}} \\
& \times f_{n}\left(\bar{R}_{A B C}\right),
\end{aligned}
$$


where $C_{9}^{A B C}$ is the corresponding $n$ th-order interatomic dispersion coefficient (approximated by $C_{9}^{A B C} \approx-\sqrt{C_{6}^{A B} C_{6}^{B C} C_{6}^{A C}}$ ) and $\theta_{i}$ are the internal angles of the triangle formed by the length of the sides $R_{A B}-R_{B C}-R_{A C}$. The damping function $f_{n}$ also holds a similar form to the ZD one used in Eq. (6) with $\bar{R}_{A B C}$ the geometric mean of $R_{A B}, R_{B C}$, and $R_{A C}$. Note that this expression takes approximately into account the screening interactions between a given pair of atoms, $A$ and $B$, by a polarizable one $C$. Very recently, the three-body contribution to the lattice energy in the benzene crystal was accurately obtained by ab initio calculations. ${ }^{64}$ The authors have shown that previous estimates in the literature had overemphasized their strength as compared with the latter reference data, and that includes approximate (and non-electronic) expression (8). However, due to the coronene size and the needed trade-off between accuracy and cost, we will rely on this approximate form to (at least qualitatively) estimate the three-body contribution in this case.

Using this scheme, the binding or interaction energy of every pair (dimer) of molecules (the supermolecular complex) within the crystalline form is composed by several terms

$$
\begin{aligned}
\Delta E_{\text {int }}= & E_{D F T-D 3}(\text { dimer })-2 E_{D F T-D 3}(\text { monomer }) \\
= & E_{D F T}(\text { dimer })+E_{\mathrm{D} 3}(A, B)(\text { dimer }) \\
& +E_{\mathrm{D} 3}(A, B, C)(\text { dimer }) \\
& -2\left[E_{D F T}(\text { monomer })+E_{\mathrm{D} 3}(A, B) \text { (monomer }\right) \\
& \left.\left.+E_{\mathrm{D} 3}(A, B, C) \text { (monomer }\right)\right]
\end{aligned}
$$

approximating thus as much as possible the effects contained in Eqs. (4) and (5).

\section{The DFT-NL method}

A different route to incorporate the long-range dispersion interactions between electronic particles uses the non-local exchange-correlation functional dubbed as VV10 ${ }^{65}$ which has the form

$$
E_{\mathrm{D}} \approx E_{x c}^{\mathrm{NL}}=\int d \mathbf{r} \rho(\mathbf{r})\left[\beta(b)+\frac{1}{2} \int d \mathbf{r}^{\prime} \rho\left(\mathbf{r}^{\prime}\right) \Phi\left(\mathbf{r}, \mathbf{r}^{\prime}\right)\right],
$$

with the function $\Phi\left(\mathbf{r}, \mathbf{r}^{\prime}\right)$ coupling the calculated electronic densities, $\rho(\mathbf{r})$ and $\rho\left(\mathbf{r}^{\prime}\right)$, at two different sampled points of the electronic coordinates space. The parameter $b$ entering into the expression plays an attenuation role and can be also used to couple any standard exchange-correlation functional with this correction, as it has been recently done for a wide variety of functionals, ${ }^{66-68}$ giving thus a final interacting energy for every pair of non-covalently bound molecules as

$$
\begin{aligned}
\Delta E_{\text {int }}= & E_{D F T-N L}(\text { dimer })-2 E_{D F T-N L}(\text { monomer }) \\
= & E_{D F T}(\text { dimer })+E_{x c}^{\mathrm{NL}}(\text { dimer }) \\
& -2\left[E_{D F T}(\text { monomer })+E_{x c}^{\mathrm{NL}}(\text { monomer })\right] .
\end{aligned}
$$

\section{Technical details}

The ORCA quantum-chemical package ${ }^{69}$ was employed for all the calculations reported in this section. We will use both schemes, DFT-D3 and DFT-NL, with the hierarchy of BLYP, B3LYP, and B2-PLYP functionals, corresponding to pure, hybrid, and double-hybrid models, respectively. ${ }^{70-73}$ All the underlying (specific for dispersion) parameters are gathered in Table I. Other technical details are presented next. The large (very large) def2-TZVP (def2-QZVP) basis sets will be thoroughly used here, which allows us to reasonably discard (at any

\begin{tabular}{|c|c|c|c|c|c|c|c|c|}
\hline & & \multirow[b]{2}{*}{$m_{i}{ }^{\mathrm{a}}$} & \multicolumn{2}{|c|}{ BLYP-D3(ZD) } & \multicolumn{2}{|c|}{ B3LYP-D3(ZD) } & \multicolumn{2}{|c|}{ B2-PLYP-D3(ZD) } \\
\hline & & & def2-TZVP & def2-QZVP & def2-TZVP & def2-QZVP & def2-TZVP & def2-QZVP \\
\hline & $(\mathrm{a} / 2, \mathrm{~b} / 2,0)$ & 4 & -17.00 & -16.53 & -16.24 & -16.07 & -16.73 & -16.73 \\
\hline & $(\mathrm{a} / 2, \mathrm{~b} / 2, \mathrm{c})$ & 4 & -8.93 & -8.62 & -8.76 & -8.52 & -8.62 & -8.45 \\
\hline & $(-\mathrm{a} / 2, \mathrm{~b} / 2, \mathrm{c})$ & 4 & -0.16 & -0.16 & -0.14 & -0.13 & -0.18 & -0.30 \\
\hline & $(\mathrm{a}, 0,0)$ & 2 & -0.10 & -0.09 & -0.10 & -0.04 & -0.14 & -0.11 \\
\hline & $(0, b, 0)$ & 2 & -78.27 & -76.84 & -69.96 & -68.20 & -84.52 & -78.12 \\
\hline & $(a, b, c)$ & 4 & -0.20 & -0.19 & -0.19 & -0.17 & -0.22 & -0.26 \\
\hline & $(a, b,-c)$ & 4 & -0.02 & -0.02 & -0.02 & -0.03 & -0.02 & -0.01 \\
\hline & $(a, b, 0)$ & 4 & -0.16 & -0.15 & -0.16 & -0.11 & -0.20 & -0.18 \\
\hline Cohesive energy ${ }^{\mathrm{b}}$ & & & 158.4 & 154.5 & 145.9 & 143.3 & 162.0 & 155.2 \\
\hline
\end{tabular}
level) the Basis Set Superposition Error (BSSE) expected for interaction energies of these non-covalently bound coronene pairs. The computational effort was significantly reduced by (i) invoking the "resolution of the identity" (RI) ${ }^{74}$ algorithm for fitting the Coulomb and correlation two-electron integrals via the corresponding matching auxiliary basis sets, ${ }^{75}$ and

TABLE II. Interaction energies (in $\mathrm{kJ} / \mathrm{mol}$ ) for the set of coronene dimers belonging to the first and second coordination spheres, calculated at the DFT-D3 level using different functionals. The corresponding derived cohesive energies are also given.

${ }^{a}$ Number of symmetry-related pairs.

${ }^{\mathrm{b}}$ Using this counting method, one needs to divide the total result by two (see Ref. 78). 

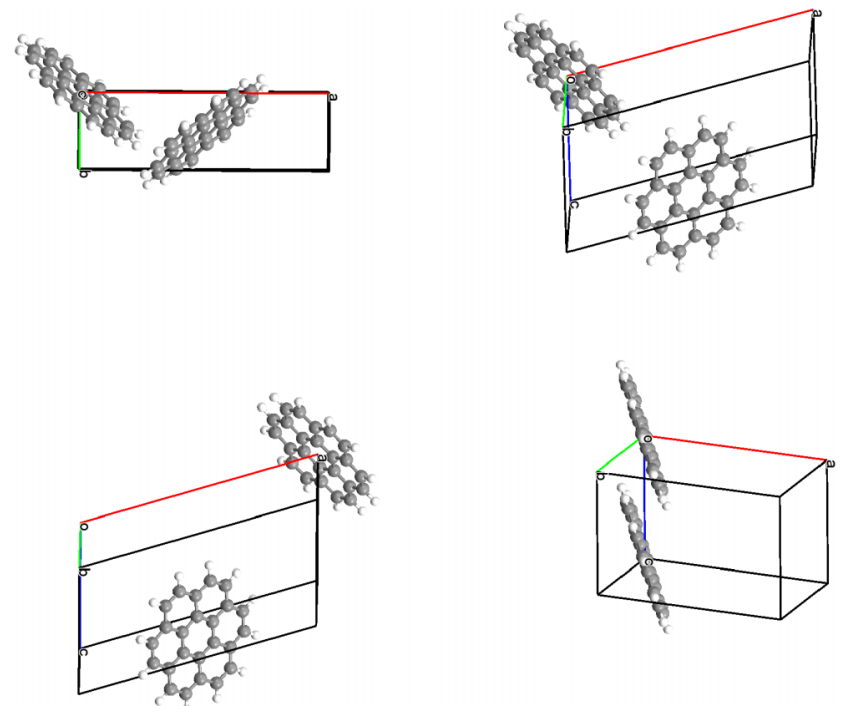

FIG. 3. Interacting dimers in the first coordination shell (from top to bottom and from left to right) as extracted from the crystalline structure: $(\mathrm{a} / 2, \mathrm{~b} / 2,0)$; $(\mathrm{a} / 2, \mathrm{~b} / 2, \mathrm{c}) ;(-\mathrm{a} / 2, \mathrm{~b} / 2, \mathrm{c}) ;$ and $(\mathrm{c}, 0,0)$.

(ii) employing the "chain-of-spheres" $(\operatorname{COSX})^{76}$ algorithm for solving the exchange integrals. Finally, to reduce as much as possible the numerical errors, the thresholds for integration grids and the convergence criteria of self-consistent calculations and optimizations were always made tighter than defaults. When (sometimes severe) convergence problems arise with the def2-QZVP basis set, the Newton-Raphson algorithm had to be turned on.

\section{B. Results and discussion}

Table II lists the DFT-D3 calculated $\Delta E_{\text {int }}$ values of the complex as obtained from Eq. (9), for each of the interacting dimers belonging to the first and second coordination shells depicted in Figures 3 and 4, respectively. Further consideration of additional shells is not expected to have a significant impact on the results, due to the well-known $R^{-6(8)}$ decay of these interactions and the small value for the interaction energies (much less than $1 \mathrm{~kJ} / \mathrm{mol}$ ) obtained for the dimers separated by the largest distances. ${ }^{77}$ The lattice or cohesive energy can then be estimated by multiplying each of the interaction energies by the number $\left(m_{i}\right)$ of symmetry-related pairs, thus giving $E_{\text {cohesive }}=-m_{i} \sum_{i} \Delta E_{\text {int }}^{i}$. Note that the final value is again made positive to compare with experimental sublimation energies, and that the result has to be divided by two as a consequence of the counting method, see Ref. 78 for more details.

First of all, comparing the DFT-based results for $E_{\text {cohesive }}$ with the def2-TZVP and def2-QZVP basis sets, we note the moderate influence of the basis set size for the BLYP and B3LYP functionals. On the other hand, for B2-PLYP, it appears to be slightly more pronounced due to the influence of the perturbative part, which is known to converge slower than the rest of terms. Extending the basis set from def2TZVP to def2-QZVP decreases the values in $3-4 \mathrm{~kJ} / \mathrm{mol}$ for BLYP or B3LYP, and up to $7 \mathrm{~kJ} / \mathrm{mol}$ in the case of the double-hybrid B2-PLYP case. We also note that the latter functional, even without the -D3 correction for dispersion, is able to render bound dimers in all cases,
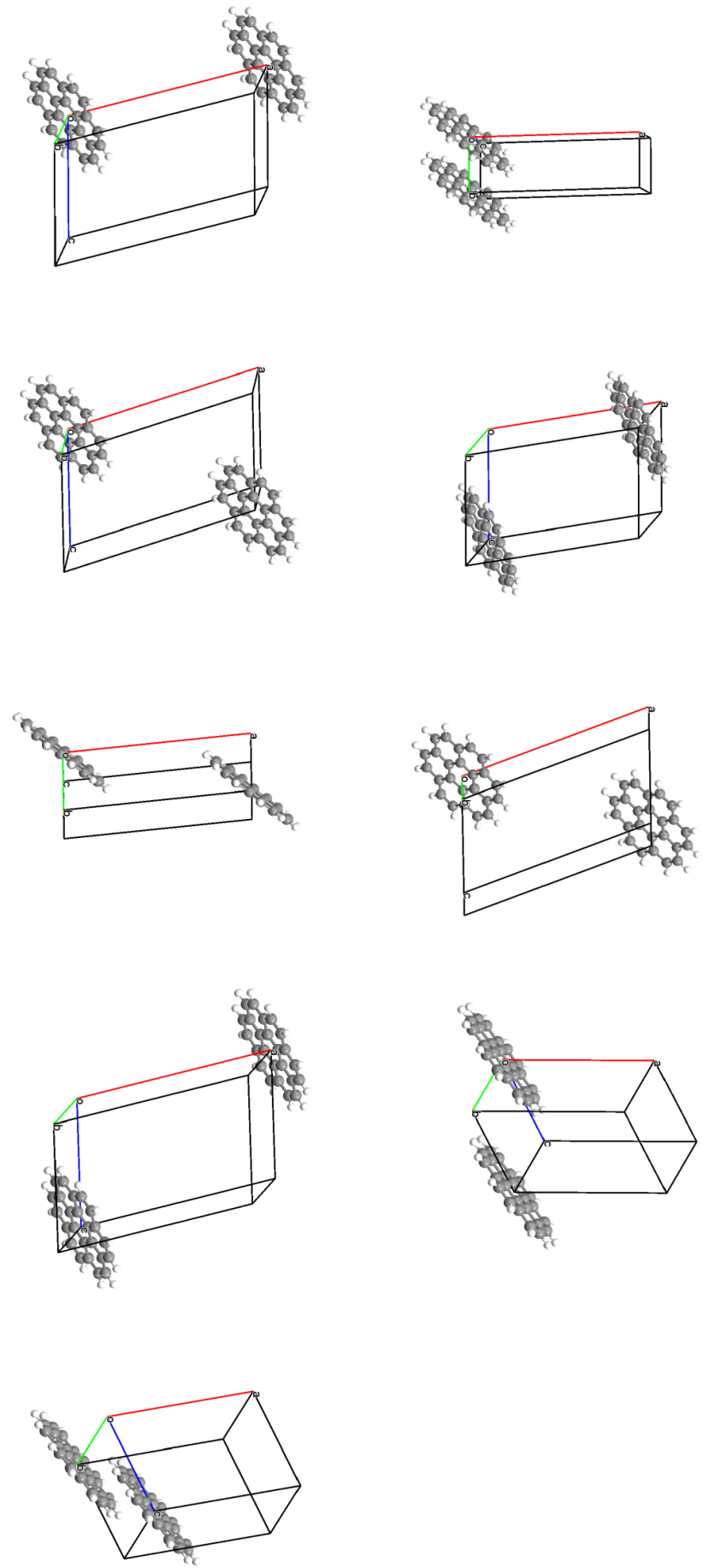

FIG. 4. Interacting dimers in the second coordination shell (from top to bottom and from left to right) as extracted from the crystalline structure: $(\mathrm{a}, 0,0) ;(0, \mathrm{~b}, 0) ;(\mathrm{a}, \mathrm{b}, \mathrm{c}) ;(\mathrm{a}, \mathrm{b},-\mathrm{c}) ;(\mathrm{a}, \mathrm{b}, 0) ;(\mathrm{a}, 0, \mathrm{c}) ;(\mathrm{a}, 0,-\mathrm{c}) ;(0, \mathrm{~b}, \mathrm{c}) ;(0, \mathrm{~b},-\mathrm{c})$.

while semilocal pure (BLYP) or hybrid (B3LYP) functionals completely neglect dispersion interactions, giving unbound dimers. However, dispersion-uncorrected (dispersion-corrected) values of $E_{\text {cohesive }}$ are severely underestimated by more than $50 \%$ (slightly overestimated) with respect to the experimental estimate in this case. Previous applications of the B2PLYP functional to other molecular crystals, such as urea, formamide, or ammonia, led to an underestimation of around $30 \%$ with respect to experimental estimates, ${ }^{79}$ although for those crystals, dispersion is expected to be of less importance 
than electrostatics interactions. Actually, since the B2-PLYP functional includes a weighted perturbative-like part, based on the second-order Möller-Plesset expression (MP2) but calculated with slightly different orbitals, the overestimation found resembles that suffered by the MP2 method itself. ${ }^{80-82}$

Hence, the -D3 correction plays a key role for all DFTbased schemes since, broadly speaking, it can be seen how the DFT-D3/def2-QZVP final calculated values oscillate in a narrow range (see Figure 5) between 143 and $162 \mathrm{~kJ} / \mathrm{mol}$ and are thus within the accuracy limits considering that the experimental data lie in the range of 139-156 kJ/mol, being also above the threshold given by the experimental vaporization energy of $139 \mathrm{~kJ} / \mathrm{mol}$. Finally, recovering the previous BLYP-D2/MTCP calculated value of $162 \mathrm{~kJ} / \mathrm{mol}$, and comparing it with the BLYP-D3(ZD)/def2-QZVP value of $154 \mathrm{~kJ} / \mathrm{mol}$, one can guess the influence of the -D3 expression with respect to the (more approximate) -D2 correction, roughly indicating the force of dipole-quadrupole interactions together with the three-body correction in the dispersion correction.

To further investigate the relative weight of the different energy contributions to the cohesive energy, we recast Eq. (9) for the BLYP-D3(ZD)/def2-QZVP method taken as example in the following way:

$$
\begin{aligned}
E_{\text {cohesive }}= & -m_{i} \sum_{i} \Delta E_{\text {int }}^{i} \\
= & -m_{i}\left\{\sum_{i}\left[\Delta E_{D F T}(\text { dimer })-2 E_{D F T}(\text { monomer })\right]\right. \\
& +\sum_{i}\left[\Delta E_{\mathrm{D} 3}^{C_{6} / R^{6}}(A, B)(\text { dimer })-2 E_{\mathrm{D} 3}^{C_{6} / R^{6}}(A, B)(\text { monomer })\right] \\
& +\sum_{i}\left[\Delta E_{\mathrm{D} 3}^{C_{8} / R^{8}}(A, B)(\text { dimer })-2 E_{\mathrm{D} 3}^{C_{8} / R^{8}}(A, B)(\text { monomer })\right] \\
& \left.+\sum_{i}\left[\Delta E_{\mathrm{D} 3}(A, B, C)(\text { dimer })-2 E_{\mathrm{D} 3}(A, B, C)(\text { monomer })\right]\right\} \\
= & -74.9+132.6+102.9-6.03=154.6 \mathrm{~kJ} / \mathrm{mol},
\end{aligned}
$$

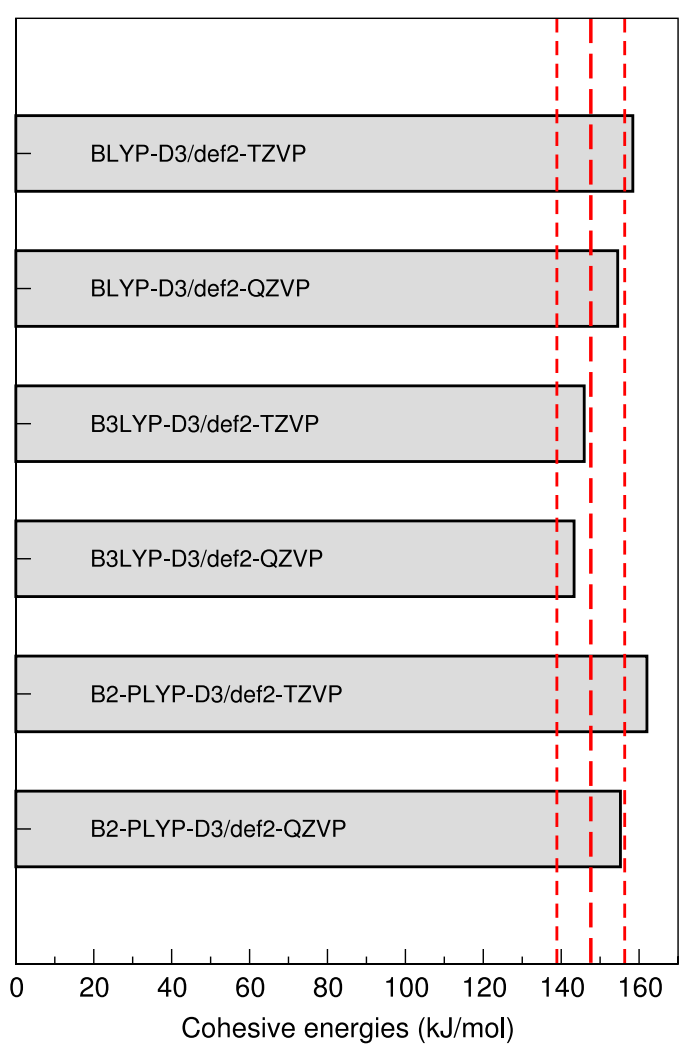

FIG. 5. Theoretical estimates of cohesive energies by DFT-D3 methods. The experimental sublimation enthalpy value (dashed red line) of $142.6 \mathrm{~kJ} / \mathrm{mol}$ and its average experimental uncertainty $( \pm 8.7 \mathrm{~kJ} / \mathrm{mol})$ are also included after adding the corresponding $2 R T$ correction. as it appears in Table II under the corresponding BLYPD3(ZD)/def2-QZVP heading. We easily see the key role played by all dispersion interactions, counterbalancing efficiently the unbound structures found at the dispersion-uncorrected BLYP/def2-QZVP level. Note that the second and third lines of Eq. (13) correspond to the $-s_{6} \sum_{B>A}^{\text {atom pairs }} \frac{C_{6}^{A B}}{R_{A B}^{6}} f_{6}\left(R_{A B}\right)$ and $-s_{8} \sum_{B>A}^{\text {atom pairs }} \frac{C_{8}^{A B}}{R_{A B}^{8}} f_{8}\left(R_{A B}\right)$ energy terms, respectively, and that the three-body term is repulsive and non-negligible if one seeks the greatest possible accuracy.

We discuss now the DFT-NL results (see Table III) calculated only with the def2-TZVP basis set, since conclusions are not expected to largely differ upon further basis set extension. We also know that (in the worst possible scenario) going from the B2-PLYP/def2-TZVP to the B2-PLYP/def2-QZVP decreases the cohesive energy by around $7 \mathrm{~kJ} / \mathrm{mol}$ (from 58.1 to $51.3 \mathrm{~kJ} / \mathrm{mol}$ ), yet the $-\mathrm{NL}$ correction is known to saturate already with the triple-zeta basis sets and will be thus less affected. Whereas the BLYP-NL and B3LYP-NL values are close to each other, 150 and $142 \mathrm{~kJ} / \mathrm{mol}$, respectively, and close in accuracy to the experimental limits, the B2-PLYPNL method seems to slightly overestimate the strength of the dispersion interaction, which in particular affects the dimers contributing the most to the $E_{\text {cohesive }}$ value, rendering a value of $178 \mathrm{~kJ} / \mathrm{mol}$. As a matter of example, the interaction energy of the $(0, \mathrm{~b}, 0)$ dimer is calculated at this level to be $-97.8 \mathrm{~kJ} / \mathrm{mol}$, and thus overestimated by $13-28 \mathrm{~kJ} / \mathrm{mol}$ with respect to any other DFT-D3 or DFT-NL method (with the same def2-TZVP basis set). This might point to the need of a larger (more 
TABLE III. Interaction energies (in $\mathrm{kJ} / \mathrm{mol}$ ) for the set of coronene dimers belonging to the first and second coordination spheres, calculated at the DFT-NL level using different functionals. The corresponding derived cohesive energies are also given.

\begin{tabular}{lccccc}
\hline \hline & & & $\begin{array}{c}\text { BLYP-NL } \\
\text { def2-TZVP }\end{array}$ & $\begin{array}{c}\text { B3LYP-NL } \\
\text { def2-TZVP }\end{array}$ & $\begin{array}{c}\text { B2-PLYP-NL } \\
\text { def2-TZVP }\end{array}$ \\
\hline 1st-shell & & $m_{i}{ }^{a}$ & & & \\
& $(\mathrm{a} / 2, \mathrm{~b} / 2,0)$ & 4 & -15.23 & -14.59 & -17.90 \\
& $(\mathrm{a} / 2, \mathrm{~b} / 2, \mathrm{c})$ & 4 & -7.04 & -6.99 & -8.71 \\
& $(-\mathrm{a} / 2, \mathrm{~b} / 2, \mathrm{c})$ & 4 & -0.16 & -0.15 & -0.24 \\
& $(0,0, \mathrm{c})$ & 2 & -13.40 & -13.55 & -16.31 \\
& & & & -0.20 \\
& $(\mathrm{a}, 0,0)$ & 2 & -0.15 & -0.16 & -97.78 \\
& $(0, \mathrm{~b}, 0)$ & 2 & -83.35 & -76.57 & -0.25 \\
& $(\mathrm{a}, \mathrm{b}, \mathrm{c})$ & 4 & -0.17 & -0.17 & -0.03 \\
& $(\mathrm{a}, \mathrm{b},-\mathrm{c})$ & 4 & -0.02 & -0.03 & -0.23 \\
& $(\mathrm{a}, \mathrm{b}, 0)$ & 4 & -0.17 & -0.18 & -0.34 \\
& $(\mathrm{a}, 0, \mathrm{c})$ & 2 & -0.24 & -0.26 & -0.04 \\
& $(\mathrm{a}, 0,-\mathrm{c})$ & 2 & -0.03 & -0.04 & -2.83 \\
& $(0, \mathrm{~b}, \mathrm{c})$ & 2 & -2.09 & -2.15 & -6.23 \\
& $(0, \mathrm{~b},-\mathrm{c})$ & 2 & -5.02 & -4.69 & 178.5 \\
\hline \hline
\end{tabular}

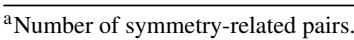

${ }^{\mathrm{b}}$ Using this counting method, one needs to divide the total result by two (see Ref. 78).

repulsive) value of the $b$ parameter in Eq. (10) and/or to the lack of many-body contributions in the DFT-NL calculations, as it is exemplified by the approximate $E_{\mathrm{D} 3}(A, B, C)$ term in DFT-D3 scheme. Note that the value of the $b$ parameter was derived after its careful fitting to minimize as much as possible the energy deviations with respect to accurate values of the complexes within the S22 and S66 databases, widely used as benchmarks and composed of a diversity of non-covalently bound complexes. ${ }^{80,83}$

Thus, to further investigate the coupling of double-hybrid density functionals with the non-local correction given by Eq. (10), using the def2-TZVP basis set, we compare the value of $E_{\text {cohesive }}$ provided by the parameter-free PBE0-DH-NL method $^{84}(177 \mathrm{~kJ} / \mathrm{mol})$ to that yielded by the corresponding PBE0-DH-D2 ${ }^{85}$ one $(198 \mathrm{~kJ} / \mathrm{mol})$. This is about the same degree of overestimation observed between B2-PLYP-D3(ZD) and B2-PLYP-NL: 162 and $178 \mathrm{~kJ} / \mathrm{mol}$, respectively. Therefore, we hypothesize at this stage about the importance in extended systems of many-body (mainly three-body) contributions to the dispersion contribution, as calculated by Eq. (8). They can also be incorporated into the DFT-NL theory as it has been shown recently. ${ }^{86}$ By adding this quantity, the values for $E_{\text {cohesive }}$ now amount to 144,136 , and $172 \mathrm{~kJ} / \mathrm{mol}$, at the BLYP-NL, B3LYP-NL, and B2-PLYP-NL levels, respectively, bringing the DFT-NL results closer to the experimental value.

Upon inspection of Tables II and III, it can be immediately seen how the $(0, b, 0)$ dimer, which has been repeatedly studied in the literature by different theoretical methods, ${ }^{87-92}$ is the structure contributing the most to the stability of the samples. We would like to emphasize how this energy gain is known to govern many interesting electronic properties of coronene samples, such as the anisotropic charge mobility values in organic electronic devices ${ }^{93,94}$ or the thermochemistry of their spontaneous self-aggregation. ${ }^{95,96}$

We can also see from Tables II and III that the interaction energy of the $(0, \mathrm{~b}, 0)$ dimer ranges from $-68 \mathrm{~kJ} / \mathrm{mol}$
(B3LYP-D3/def2-QZVP) to -98 kJ/mol (B2-PLYP-NL/def2TZVP). We want to note also that the average value of -79 $\mathrm{kJ} / \mathrm{mol}$ is close to that provided by the methods yielding very accurate estimates of total cohesive energies: BLYP-D3/def2QZVP and B2-PLYP-D3/def2-QZVP. The contribution (in percentage) of the energy stability of this dimer to the final $E_{\text {cohesive }}$ values as a function of the method employed using the larger def2-QZVP basis set varies as follows: 49.7\%, $47.6 \%$, and $50.3 \%$, for the BLYP-D3, B3LYP-D3, and B2PLYP-D3 methods, respectively. Slightly larger contributions (around 55\%) are obtained at the DFT-NL level, in agreement with the overestimation also found for total cohesive energies. Nonetheless, and independently of the small differences found between the methods employed, it is easily observed the leading role played by this dimer for the final values. This importance totally justifies the inclusion of this dimer in databases for benchmarking, as it was recently done in the L7 dataset (named there as $\mathrm{C} 2 \mathrm{C} 2 \mathrm{PD})$ of large supramolecular complexes. ${ }^{97}$

\section{CONCLUSIONS}

We have addressed here the role played by dispersion forces for the energy stability of crystalline coronene. We have analyzed the results following a top-down approach and comparing them with experimental available values. These forces control the nanoscopic structure of organic materials and are thus of the most interest.

First, we have found small numerical differences of only $1 \mathrm{~kJ} / \mathrm{mol}$ (at the DFT-D2 level) due to the use of different pseudopotentials in periodic boundary calculations. The use of different basis sets in non-periodic calculations involves larger numerical differences around 3-4 (7) $\mathrm{kJ} / \mathrm{mol}$ for pure and hybrid (double-hybrid) functionals.

Interestingly, we have disclosed (at the DFT-D3 level) the large role played by dipole-quadrupole interactions, showing 
that this stabilizing effect is of utmost importance irrespective of other effects. The three-body contributions to the dispersion energy, which are beyond interatomic pair interactions, destabilize the cohesive energy (again at the DFT-D3 level) by $6 \mathrm{~kJ} / \mathrm{mol}$.

Furthermore, after comparing the DFT-NL and DFT-D3 approaches, with the three-body contribution added, one also sees a difference of roughly $10 \mathrm{~kJ} / \mathrm{mol}$, with the latter over- (using BLYP and B3LYP) or underestimating (using B2-PLYP) the values provided by the former approach.

The calculations of the cohesive energy performed in this investigation ranged between 133 and $172 \mathrm{~kJ} / \mathrm{mol}$, and are in reasonable agreement with an (averaged) experimental reference value of $143 \mathrm{~kJ} / \mathrm{mol}$, showing the predictive capacity of dispersion-corrected theoretical calculations in molecular and condensed matter physics.

However, generally speaking, considering the timing invested in the calculations, and the trade off between accuracy and computational cost always needed, the DFT-D3 or DFT-NL methods with pure functionals (i.e., BLYP here) and with the def2-TZVP (or equivalent) basis set appear as quite successful.

\section{ACKNOWLEDGMENTS}

Financial support by the "Ministerio de Economía y Competitividad" of Spain and the "European Regional Development Fund" through Project No. CTQ2011-27253. The work in Mons was supported by the "Programme d'Excellence de la Région Wallonne” (OPTI2MAT project) and FNRS-FRFC.

${ }^{1}$ G. R. Desiraju and A. Gavezzotti, J. Chem. Soc., Chem. Commun. 1989, 621.

${ }^{2}$ G. R. Desiraju and A. Gavezzotti, Acta Crystallogr., Sect. B: Struct. Sci. 45, 473 (1989).

${ }^{3}$ A. Tkatchenko, R. A. DiStasio, R. Car, and M. Scheffler, Phys. Rev. Lett. 108, 236402 (2012).

${ }^{4}$ R. A. DiStasio, A. O. von Lilienfeld, and A. Tkatchenko, Proc. Natl. Acad. Sci. U. S. A. 109, 14791 (2012).

${ }^{5}$ A. Tkatchenko, A. Ambrosetti, and R. A. DiStasio, J. Chem. Phys. 138, 074106 (2013).

${ }^{6}$ R. A. DiStasio, V. V. Gobre, and A. Tkatchenko, J. Phys.: Condens. Matter 26, 213202 (2014)

${ }^{7}$ G. J. O. Beran, S. Wen, K. Nanda, Y. Huang, and Y. Heit, Top. Curr. Chem. 345, 59 (2014).

${ }^{8}$ J. F. Dobson, Int. J. Quantum Chem. 114, 1157 (2014).

${ }^{9}$ A. Otero-de-la-Roza and E. R. Johnson, J. Chem. Phys. 137, 054103 (2012).

${ }^{10}$ A. M. Reilly and A. Tkatchenko, J. Phys. Chem. Lett. 4, 1028 (2013).

${ }^{11}$ J. G. Branderburg and S. Grimme, Top. Curr. Chem. 345, 1 (2014).

${ }^{12}$ J. G. Branderburg and S. Grimme, J. Phys. Chem. Lett. 5, 1785 (2014).

${ }^{13}$ J. Moellmann and S. Grimme, J. Phys. Chem. C 118, 7615 (2014).

${ }^{14}$ M. D. King, T. N. Blanton, and T. M. Korter, Phys. Chem. Chem. Phys. 14, 1113 (2012).

${ }^{15}$ J. Binns, M. R. Healy, S. Parsons, and C. A. Morrison, Acta Crystallogr., Sect. B: Struct. Sci., Cryst. Eng. Mater. 70, 259 (2014).

${ }^{16}$ L. Maschio, B. Civalleri, P. Ugliengo, and A. Gavezzotti, J. Phys. Chem. A 115, 11179 (2011).

${ }^{17}$ C. R. Taylor, P. J. Bygrave, J. N. Hart, N. L. Allan, and F. R. Manby, Phys. Chem. Chem. Phys. 14, 7739 (2012).

${ }^{18}$ S. Wen and G. J. O. Beran, Cryst. Growth Des. 12, 2169 (2012).

${ }^{19}$ A. M. Reilly and A. Tkatchenko, Phys. Rev. Lett. 113, 055702 (2014).

${ }^{20}$ S. Wen and G. J. O. Beran, J. Chem. Theory Comput. 8, 2698 (2012).

${ }^{21}$ K. Hongo, M. A. Watson, R. S. Sánchez-Carrera, T. Itaka, and A. AspuruGuzik, J. Phys. Chem. Lett. 1, 1789 (2010).

${ }^{22}$ K. E. Riley, M. Pitoňák, P. Jurečka, and P. Hobza, Chem. Rev. 110, 5023 (2010).

${ }^{23}$ P. Hobza, Annu. Rep. Prog. Chem., Sect. C: Phys. Chem. 107, 148 (2011).
${ }^{24}$ S. Grimme, WIREs: Comput. Mol. Sci. 1, 211 (2011).

${ }^{25}$ H. Eshuis, J. E. Bates, and F. Furche, Theor. Chem. Acc. 131, 1084 (2012).

${ }^{26}$ M. P. Waller, H. Kruse, C. Mück-Lichtenfeld, and S. Grimme, Chem. Soc. Rev. 41, 3119 (2012).

${ }^{27}$ J. Klimeš and A. Michaelides, J. Chem. Phys. 137, 120901 (2012).

${ }^{28}$ J. F. Dobson and T. Gould, J. Phys.: Condens. Matter 24, 073201 (2012).

${ }^{29}$ S. Ehrlich, J. Moellmann, and S. Grimme, Acc. Chem. Res. 46, 916 (2013).

${ }^{30}$ S. E. Huber and M. Probst, Int. J. Mass Spectrom. 365, 248 (2014).

${ }^{31}$ B. Schatschneider, S. Monaco, J.-J. Liang, and A. Tkatchenko, J. Phys. Chem. C 118, 19964 (2014).

${ }^{32}$ N. Mardirossian and M. Head-Gordon, Phys. Chem. Chem. Phys. 16, 9904 (2014).

${ }^{33}$ H. Ruuska and T. A. Pakkanen, J. Phys. Chem. B 105, 9541 (2001).

${ }^{34}$ J. Ma, A. Michaelides, and D. Alfe, J. Chem. Phys. 134, 134701 (2011).

${ }^{35}$ S. E. Wheeler, CrystEngComm 14, 6140 (2012).

${ }^{36}$ J. S. Chickos and W. E. Acree, Jr., J. Phys. Chem. Ref. Data 31, 537 (2002).

${ }^{37}$ Y. Huang, Y. Shao, and G. J. O. Beran, J. Chem. Phys. 138, 224112 (2013).

${ }^{38}$ M. V. Roux, M. Temprano, J. S. Chickos, and Y. Nagano, J. Phys. Chem. Ref. Data 37, 1855 (2008).

${ }^{39}$ J. K. Fawcett and J. Trotter, Proc. R. Soc. London, Ser. A 289, 366 (1966).

${ }^{40}$ C. F. Macrae, I. J. Bruno, J. A. Chisholm, P. R. Edgington, P. McCabe, E. Pidcock, L. Rodríguez-Monge, R. Taylor, J. van de Streek, and P. A. Wood, J. Appl. Crystallogr. 41, 466 (2008).

${ }^{41}$ A. M. Reilly and A. Tkatchenko, J. Chem. Phys. 139, 024705 (2013).

${ }^{42}$ L. Kronik and A. Tkatchenko, Acc. Chem. Res. 47, 3208 (2014).

${ }^{43}$ F. Bottin, S. Leroux, A. Knyazev, and G. Zerath, Comput. Mater. Sci. 42, 329 (2008).

${ }^{44}$ X. Gonze, B. Amadon, P.-M. Anglade, J.-M. Beuken, F. Bottin, P. Boulanger, F. Bruneval, D. Caliste, R. Caracas, M. Cote, T. Deutsch, L. Genovese, Ph. Ghosez, M. Giantomassi, S. Goedecker, D. R. Hamann, P. Hermet, F. Jollet, G. Jomard, S. Leroux, M. Mancini, S. Mazevet, M. J. T. Oliveira, G. Onida, Y. Pouillon, T. Rangel, G.-M. Rignanese, D. Sangalli, R. Shaltaf, M. Torrent, M. J. Verstraete, G. Zerah, and J. W. Zwanziger, Comput. Phys. Commun. 180, 2582 (2009).

${ }^{45}$ M. A. L. Marques, M. J. T. Oliveira, and T. Burns, Comput. Phys. Commun. 183, 2227 (2012).

${ }^{46}$ S. Grimme, J. Comput. Chem. 27, 1787 (2006).

${ }^{47}$ P. Jurečka, J. Černý, P. Hobza, and D. R. Salahub, J. Comput. Chem. 28, 555 (2007).

${ }^{48}$ J. P. Perdew, M. Ernzerhof, and K. Burke, J. Chem. Phys. 105, 9982 (1996).

${ }^{49}$ A. D. Becke, Phys. Rev. A 38, 3098 (1988).

${ }^{50}$ C. Lee, W. Yang, and R. G. Parr, Phys. Rev. B 37, 785 (1988).

${ }^{51}$ I. Fedorov, Y. Zhuravlev, and V. Berveno, Phys. Status Solidi B 249, 1438 (2012).

${ }^{52}$ M. Steinmetz, A. Hansen, S. Ehrlich, T. Risthaus, and S. Grimme, Top. Curr. Chem. 128, 543 (2014).

${ }^{53}$ F. Kannemann and A. D. Becke, J. Chem. Theory Comput. 5, 719 (2009).

${ }^{54}$ I. Fedorov, Y. Zhuravlev, and V. Berveno, Phys. Chem. Chem. Phys. 13, 5679 (2011).

${ }^{55}$ I. Fedorov, Y. Zhuravlev, and V. Berveno, J. Chem. Phys. 138, 094509 (2013).

${ }^{56}$ J. Yang, W. Hu, D. Usvyat, D. Matthews, M. Schütz, and G. K.-L. Chan, Science 345, 640 (2014)

${ }^{57}$ A. J. Misquitta, in Handbook of Computational Chemistry, edited by J. Leszczynski (Springer, Netherlands, 2012).

${ }^{58}$ S. Wen, K. Nanda, Y. Huang, and G. J. O. Beran, Phys. Chem. Chem. Phys. 14, 7578 (2012).

${ }^{59}$ S. Grimme, J. Antony, S. Ehrlich, and H. Krieg, J. Chem. Phys. 132, 154104 (2010).

${ }^{60}$ E. R. Johnson and A. D. Becke, J. Chem. Phys. 123, 024101 (2005).

${ }^{61}$ E. R. Johnson and A. D. Becke, J. Chem. Phys. 124, 174104 (2006).

${ }^{62}$ S. Grimme, S. Ehrlich, and L. Goerigk, J. Comput. Chem. 32, 1456 (2011).

${ }^{63} \mathrm{O}$. A. von Lilienfeld and A. Tkatchenko, J. Chem. Phys. 132, 234109 (2010).

${ }^{64}$ M. R. Kennedy, A. R. McDonald, A. E. DePrince III, M. S. Marshall, R. Podeszwa, and C. David Sherrill, J. Chem. Phys. 140, 121104 (2014).

${ }^{65}$ O. A. Vydrov and T. Van Voorhis, J. Chem. Phys. 133, 244103 (2010).

${ }^{66}$ W. Hujo and S. Grimme, J. Chem. Theory Comput. 7, 3866 (2011).

${ }^{67}$ W. Hujo and S. Grimme, J. Chem. Theory Comput. 9, 308 (2013).

${ }^{68}$ J. Aragó, E. Ortí, and J. C. Sancho-García, J. Chem. Theory Comput. 9, 3437 (2013).

${ }^{69}$ F. Neese, WIREs: Comput. Mol. Sci. 2, 73 (2012).

${ }^{70}$ A. D. Becke, J. Chem. Phys. 98, 5648 (1993).

${ }^{71}$ V. Barone and C. Adamo, Chem. Phys. Lett. 224, 432 (1994).

${ }^{72}$ P. J. Stephens, F. J. Devlin, C. F. Chabalowski, and M. J. Frisch, J. Phys. Chem. 98, 11623 (1994). 
${ }^{73}$ S. Grimme, J. Chem. Phys. 124, 034108 (2006).

${ }^{74}$ K. Eichkorn, O. Trutler, H. Öhm, M. Häser, and R. Ahlrichs, Chem. Phys. Lett. 240, 283 (1995).

${ }^{75}$ K. Eichkorn, F. Weigend, O. Trutler, and R. Ahlrichs, Theor. Chem. Acc. 97, 119 (1997).

${ }^{76}$ F. Neese, F. Wennmohs, A. Hansen, and U. Becker, Chem. Phys. 356, 98 (2009).

${ }^{77}$ J. C. Sancho-García, J. Aragó, E. Ortí, and Y. Olivier, J. Chem. Phys. 138, 204304 (2013).

${ }^{78}$ W. B. Schweizer and J. B. Dunitz, J. Chem. Theory Comput. 2, 288 (2006).

${ }^{79}$ K. Sharkas, J. Toulouse, L. Maschio, and B. Civalleri, J. Chem. Phys. 141, 044105 (2014).

${ }^{80}$ P. Jurečka, J. Sponer, J. Černy, and P. Hobza, Phys. Chem. Chem. Phys. 8, 1985 (2006).

${ }^{81}$ S. M. Cybulski and M. L. Lytle, J. Chem. Phys. 127, 141102 (2007).

${ }^{82}$ A. Tkatchenko, R. A. DiStasio, Jr., M. Head-Gordon, and M. Schefer, J. Chem. Phys. 131, 094106 (2009).

${ }^{83}$ J. Řezáć, K. E. Riley, and P. Hobza, J. Chem. Theory Comput. 7, 2427 (2011).

${ }^{84}$ E. Brémond and C. Adamo, J. Chem. Phys. 135, 024106 (2011).

${ }^{85}$ J. C. Sancho-García, Chem. Phys. Lett. 535, 136 (2012).
${ }^{86}$ T. Risthaus and S. Grimme, J. Chem. Theory Comput. 9, 1580 (2013).

${ }^{87}$ S. Grimme, C. Mück-Lichtenfeld, and J. Antony, J. Phys. Chem. C 111, 11199 (2007).

${ }^{88}$ O. I. Obolensky, V. V. Semenikhina, A. V. Solov'yov, and W. Greiner, Int. J. Quantum. Chem. 107, 1336 (2007).

${ }^{89}$ Y. Zhao and D. G. Truhlar, J. Phys. Chem. C 112, 4061 (2008).

${ }^{90}$ I. D. Mackie and G. A. DiLabio, J. Phys. Chem. A 112, 10968 (2008).

${ }^{91}$ C. Feng, C. S. Lin, W. Fan, R. Q. Zhang, and M. A. Van Hove, J. Chem. Phys. 131, 194702 (2009).

${ }^{92}$ T. Janowski and P. Pulay, J. Am. Chem. Soc. 134, 17520 (2012).

${ }^{93}$ J. Li, Y. Liu, Y. Qian, L. Li, L. Xie, J. Shang, T. Yu, M. Yi, and W. Huang, Phys. Chem. Chem. Phys. 15, 12694 (2013).

${ }^{94}$ S. Sanyal, A. K. Manna, and S. K. Pati, J. Phys. Chem. C 117, 825 (2013).

${ }^{95}$ M. Rapacioli, F. Calvo, F. Spiegelman, C. Joblin, and D. J. Wales, J. Phys. Chem. A 109, 2487 (2005).

${ }^{96}$ L. M. da Costa, S. R. Stoyanov, S. Gusarov, P. R. Seidl, J. W. de M. Carneiro, and A. Kovalenko, J. Phys. Chem. A 118, 896 (2014).

${ }^{97}$ R. Sedlak, T. Janowski, M. Pitoňák, J. Řezáč, P. Pulay, and P. Hobza, J. Chem. Theory Comput. 9, 3364 (2013). 